\title{
Flexible IGZO thin-film transistors with liquid EGaIn gate contacts
}

\author{
Filippo Spina, Júlio C. Costa, Niko Münzenrieder \\ Flexible Electronics Laboratory, Sensor Technology Research Centre, Department of Engineering and Design, University of \\ Sussex, Falmer, Brighton, BN1 9QT, UK \\ f.spina@sussex.ac.uk
}

\section{SUMmARY AND MOTIVATION}

Flexible thin-film electronics leverage technological innovations in fields such as sensors, wearable computing and healthcare. As these systems are required to conform to non-planar surfaces, novel approaches are developed to provide stable performance under mechanical stress, and prevent crack formation. Liquid eutectic-GaIn promises the realisation of selfhealing, reconfigurable and bendable circuits. Here, a liquid EGaIn-gate thin-film transistor is fabricated and characterised. The device yielded a carrier mobility of $7.9 \mathrm{~cm}^{2} \mathrm{~V}^{-1} \mathrm{~s}^{-1}$ that increased by $0.36 \mathrm{~cm}^{2} \mathrm{~V}^{-1} \mathrm{~s}^{-1}$ when bent to a $4 \mathrm{~mm}$ radius. These results promote the integration of highly deformable liquid materials into thin-film devices.

\section{INTRODUCTION}

The conformability of flexible electronic systems enables the introduction of imperceptible and ubiquitous devices into a growing number of use cases [1]. In this regard, amorphous Indium-Gallium-Zinc-Oxide (IGZO) thin-film transistors (TFT) have been used to realise flexible electronics for deformable sensor systems used in wearable computing or biomedical applications [2, 3]. IGZO TFTs can be fabricated on various substrates and offer excellent electrical performance with a carrier mobility in the range of $\approx 15 \mathrm{~cm}^{2} \mathrm{~V}^{-1} \mathrm{~s}^{-1}$ [4]. Nevertheless, the limited ductility of polycrystalline metal thin films restricts the performance of TFTs when bent, as strain leads to the formation of cracks. This is in particular relevant for the thinner areas of these devices such as the gate contacts $[5,6]$. In this context, the self-healing and elastic properties of liquid eutectic GaIn (EGaIn) enable the implementation of this metal alloy as highly conformable electric interconnects [7]. This already led to TFT arrays supporting tensile strains up to $40 \%$ [8]. At the same time the use of this material as an integral part of active devices is a new field of research. Other liquidphase materials such as ionic liquids have only been applied as high capacitance gate dielectrics, or to dynamically dope the TFT semiconductor [9, 10]. Herein, we propose a novel approach, where liquid EGaIn is used to modulate the semiconductor channel of a flexible IGZO TFT. This approach constitutes an advancement in the realisation of semi-liquid structures for self-healing electronic circuits.

\section{DEVICE STRUCTURE AND FABRICATION}

Fig. 1a shows the structure of the transistor fabricated on a free-standing $50 \mu \mathrm{m}$ thick polyimide foil covered with $50 \mathrm{~nm}$
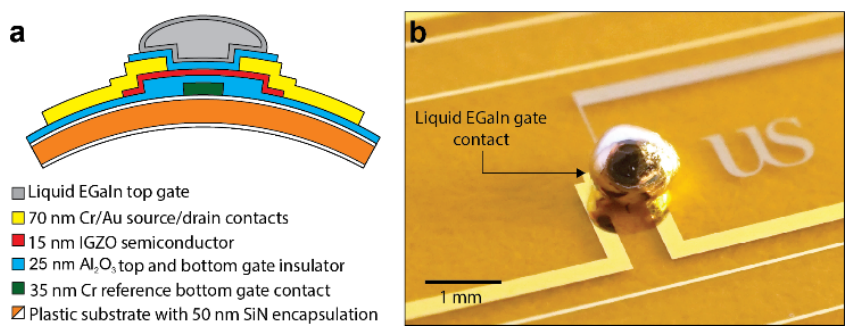

Fig. 1. Flexible IGZO thin film transistor with liquid EGaIn gate contact. a) Schematic cross-section. b) Device micrograph.

SiN. All layers were structured using standard UV lithography. The semiconductor is a $15 \mathrm{~nm}$ thick IGZO layer RF-sputtered at room temperature. It is contacted using e-beam evaporated $\mathrm{Ti} / \mathrm{Au}(10 \mathrm{~nm} / 60 \mathrm{~nm})$ source drain (S/D) contacts. The TFT width/length ratio is $560 \mu \mathrm{m} / 60 \mu \mathrm{m}$. The channel is insulated using $25 \mathrm{~nm}$ thick $\mathrm{Al}_{2} \mathrm{O}_{3}$ deposited by atomic layer deposition at $150^{\circ} \mathrm{C}$. More details on the fabrication can be found in [11]. The channel conductance is modulated by a liquid EGaIn droplet acting as top gate. This droplet was placed on the channel using a micro manipulator (Fig. 1b). The $1 \mathrm{~nm}$ thick natural gallium oxide layer covering this droplet has no significant influence on the capacitive coupling between the liquid gate and the semiconductor channel [12]. Additionally, an independent $35 \mathrm{~nm}$ Cr bottom gate was used as a reference. However, this gate has a width of only $40 \mu \mathrm{m}$, which results in a total negative S/D overlap of $20 \mu \mathrm{m}$. This negative overlap ensures that the reference and EGaIn gate will act independently on the semiconductor channel, and minimises parasitic coupling between the gates.

\section{RESUlTS AND Discussion}

The electrical characterisation of the device was carried out using a Keysight B1500A parameter analyser. All measurements were conducted in the dark at room temperature. Performance parameters were extracted from the saturation regime using the Shichman-Hodges model [13].

\section{A. Transistor performance}

Fig. 2a illustrates the capacitive coupling between the liquid EGaIn gate, as well as the reference gate with the semiconductor channel. The measured transfer and output characteristics for both configurations are presented in Figs. $2 \mathrm{~b}$ to 2e. From the reference TFT transfer curve a threshold voltage $\left(V_{t h}\right)$ of $-0.53 \mathrm{~V}$, an effective field-effect mobility $\left(\mu_{F E}\right)$ of $0.12 \mathrm{~cm}^{2} \mathrm{~V}^{-1} \mathrm{~s}^{-1}$, a subthreshold swing (SS) of 

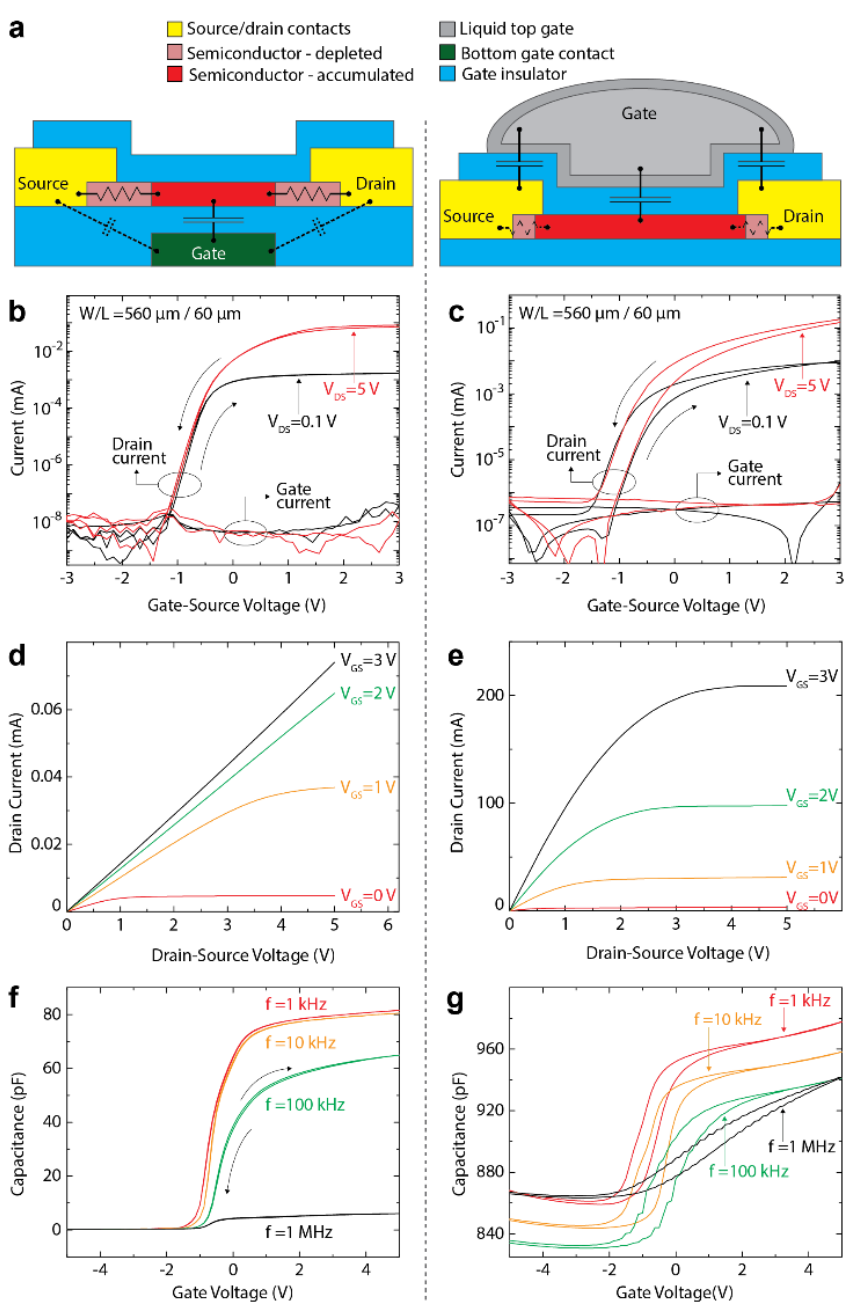

Fig. 2. DC device performance of the reference gate operation mode (left column), and the liquid EGaIn mode (right column). a) Visualisation of the coupling properties between the channel and the contacts. b-g) Transfer, output and CV (with the S/D contacts shorted, and measured for different excitation frequencies) characteristics.

$240 \mathrm{mV} /$ dec., a $I_{O N} / I_{\text {OFF }}$ current ratio $>10^{7}$, and a specific transconductance $g_{m} / \mathrm{W}\left(V_{G S}=3 V\right.$ and $\left.V_{D S}=5 \mathrm{~V}\right)$ of $6.8 \mathrm{mSm}^{-1}$ were extracted. The gate leakage current is $<10 \mathrm{pA}$ and no hysteresis is observed. As illustrated in Fig. 2a (left), the low effective mobility is the result of the negative gate-to-S/D overlaps causing no overlap capacitance (Fig. 2f) and a high contact resistance of $\approx 12 \mathrm{k} \Omega$ [14]. This is also visible in the corresponding output characteristic, which shows a maximum specific drain conductance $g_{d} / \mathrm{W}$ of $27.5 \mathrm{mSm}^{-1}$. These values result in an intrinsic transistor gain of 0.25 .

In comparison, the EGaIn-gate TFT presents a $V_{\text {th }}$ of $-0.83 \mathrm{~V}$, a $\mu_{F E}$ of $7.9 \mathrm{~cm}^{2} \mathrm{~V}^{-1} \mathrm{~s}^{-1}$, a $I_{O N} / I_{O F F}$ ratio $>10^{5}$, a specific transconductance $\left(V_{G S}=3 \mathrm{~V}\right.$ and $\left.V_{D S}=5 \mathrm{~V}\right)$ of $187.5 \mathrm{mSm}^{-1}$, and a specific output conductance of only $66 \mu \mathrm{Sm}^{-1}$, which results in an intrinsic gain of 2840 . The gate leakage current of this device is also $<10 \mathrm{pA}$, which shows that the liquid EGaIn does not deteriorate the $\mathrm{Al}_{2} \mathrm{O}_{3}$ gate insulator. The observed performance improvement for the EGaIn-gate operation mode reflects the improved capacitive coupling compared to the reference gate (Fig. 2a right). As a result, the larger overlap achieved with the droplet enables the complete
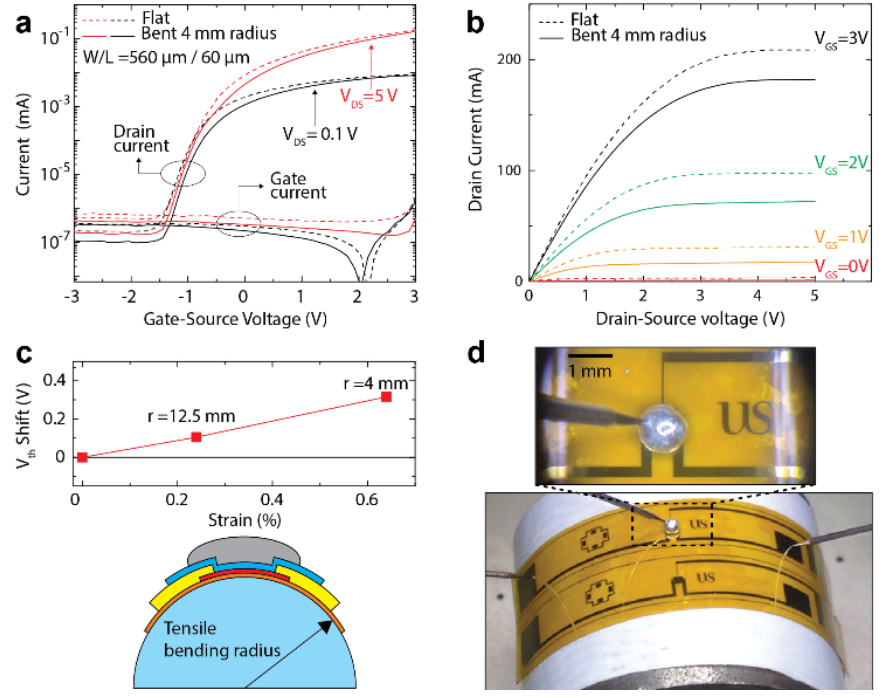

Fig. 3. a) Transfer and b) output characteristics of the flat and bent EGaIn gate TFT. c) Evolution of the threshold voltage. d) Image of the bent device.

modulation of the semiconductor channel leading to a contact resistance of $\approx 1 \mathrm{k} \Omega$. At the same time, the liquid gate configuration resulted in an increased $S S$ of $469 \mathrm{mV} / \mathrm{dec}$. and a maximum counter-clockwise hysteresis of $0.36 \mathrm{~V}$. As this is not observed for the reference gate, charge trapping at the semiconductor / gate-dielectric interface as an origin for this phenomenom is excluded and indicates that it is related to charge effects at either the gallium-oxide $/ \mathrm{Al}_{2} \mathrm{O}_{3}$ or the EGaIn / gallium-oxide interface [12]. Finally, the capacitance-voltage measurements (Figs. 2f and 2g) show that the channel capacitance $(\mathrm{f}=1 \mathrm{kHz})$ increases from $81 \mathrm{pF}$ to $109 \mathrm{pF}$ if the reference gate and EGaIn gate performances are compared. These results are in line with the geometry of the corresponding gates. Furthermore, the size of the EGaIn droplet causes an overlap capacitance of $850 \mathrm{pF}$.

\section{B. Bending}

To study the behaviour of the liquid EGaIn-gate TFT under mechanical strain, the sample was bent using rods with radii of $12.5 \mathrm{~mm}$ and $4 \mathrm{~mm}$. Figs. 3a and 3b show the transfer and output curves of the flat and bent $(4 \mathrm{~mm})$ liquid gate IGZO TFT. This corresponds to a tensile mechanical strain up to $0.64 \%$ [15]. The resulting evolution of the threshold voltage is shown in Fig. 3c. Tensile bending was applied parallel to the channel as illustrated in Figs. 3c and 3d. When bent to a $4 \mathrm{~mm}$ radius, $V_{t h}$ and $\mu_{F E}$ increased by $0.32 \mathrm{~V}$ and $0.36 \mathrm{~cm}^{2} \mathrm{~V}^{-1} \mathrm{~s}^{-1}$, which results in a $10.4 \%$ decrease of $I_{D S}$ (Fig. 3b). Comparable strain-induced shifts have previously been observed in conventional IGZO TFTs [16], in particular the $\mu_{F E}$ increase is attributed to the reduction of the effective mass under tensile strain, typical for semiconductors [17]. These measurements confirm that the TFT remains functional under strain.

In summary, a flexible amorphous oxide TFT with a liquid EGaIn gate demonstrating a mobility of $7.9 \mathrm{~cm}^{2} \mathrm{~V}^{-1} \mathrm{~s}^{-1}$, and bendability down to a radius of $4 \mathrm{~mm}$ is presented. To the best of our knowledge, this is the first partially liquid IGZO TFT. This shows the feasibility of future semi-liquid TFTs integrated in self-healing, elastic and reconfigurable electronics. 


\section{REFERENCES}

[1] A. Nathan et al., "Flexible Electronics: The Next Ubiquitous Platform," Proceedings of the IEEE, vol. 100, no. Special Centennial Issue, pp. 14861517, 2012, doi: 10.1109/jproc.2012.2190168.

[2] K. Nomura, H. Ohta, A. Takagi, T. Kamiya, M. Hirano, and H. Hosono, "Room-temperature fabrication of transparent flexible thin-film transistors using amorphous oxide semiconductors," Nature, vol. 432, no. 7016, pp. 488-92, Nov 25 2004, doi: 10.1038/nature03090.

[3] J. C. Costa, F. Spina, P. Lugoda, L. Garcia-Garcia, D. Roggen, and N. Münzenrieder, "Flexible Sensors-From Materials to Applications," Technologies, vol. 7, no. 2, p. 35, 2019, doi: 10.3390/technologies7020035.

[4] L. Petti et al., "Metal oxide semiconductor thin-film transistors for flexible electronics," Applied Physics Reviews, vol. 3, no. 2, p. 021303, 2016, doi: 10.1063/1.4953034.

[5] N. Munzenrieder et al., "Investigation of gate material ductility enables flexible a-IGZO TFTs bendable to a radius of $1.7 \mathrm{~mm}, "$ pp. 362-365, 2013, doi: 10.1109/essderc.2013.6818893.

[6] P. I. Hsu, H. Gleskova, M. Huang, Z. Suo, S. Wagner, and J. C. Sturm, "Amorphous Si TFTs on plastically deformed spherical domes," Journal of Non-Crystalline Solids, vol. 299-302, pp. 1355-1359, 2002, doi: 10.1016/s0022-3093(01)01156-5.

[7] M. D. Dickey, R. C. Chiechi, R. J. Larsen, E. A. Weiss, D. A. Weitz, and G. M. Whitesides, "Eutectic gallium-indium (EGaIn): a liquid metal alloy for the formation of stable structures in microchannels at room temperature," (in English), Advanced Functional Materials, vol. 18, no. 7, pp. 1097-1104, Apr 11 2008, doi: 10.1002/adfm.200701216.

[8] C. W. Park, J. B. Koo, C.-S. Hwang, H. Park, S. G. Im, and S.-Y. Lee, "Stretchable active matrix of oxide thin-film transistors with monolithic liquid metal interconnects," Applied Physics Express, vol. 11, no. 12, p. 126501, 2018, doi: 10.7567/apex.11.126501.

[9] P. R. Pudasaini et al., "Ionic Liquid Activation of Amorphous MetalOxide Semiconductors for Flexible Transparent Electronic Devices,"
Advanced Functional Materials, vol. 26, no. 17, pp. 2820-2825, 2016, doi: 10.1002/adfm.201505274.

[10] M. Singh et al., "The double layer capacitance of ionic liquids for electrolyte gating of $\mathrm{ZnO}$ thin film transistors and effect of gate electrodes," Journal of Materials Chemistry C, vol. 5, no. 14, pp. 35093518, 2017, doi: 10.1039/c7tc00800g.

[11] N. Munzenrieder et al., "Oxide Thin-Film Electronics on Carbon Fiber Reinforced Polymer Composite," IEEE Electron Device Letters, vol. 38, no. 8, pp. 1043-1046, 2017, doi: 10.1109/led.2017.2720258.

[12] M. R. Khan, C. B. Eaker, E. F. Bowden, and M. D. Dickey, "Giant and switchable surface activity of liquid metal via surface oxidation," Proceedings of the National Academy of Sciences, vol. 111, no. 39, pp. 14047-14051, 2014, doi: doi.org/10.1073/pnas.1412227111.

[13] H. Shichman and D. A. Hodges, "Modeling and simulation of insulatedgate field-effect transistor switching circuits," IEEE Journal of SolidState Circuits, vol. 3, no. 3, pp. 285-289, 1968, doi: 10.1109/jssc.1968.1049902.

[14] E. N. Cho, J. H. Kang, and I. Yun, "Contact resistance dependent scalingdown behavior of amorphous InGaZnO thin-film transistors," Current Applied Physics, vol. 11, no. 4, pp. 1015-1019, 2011, doi: 10.1016/j.cap.2011.01.017.

[15] H. Gleskova, S. Wagner, and Z. Suo, "a-Si:H thin film transistors after very high strain," Journal of Non-Crystalline Solids, vol. 266-269, pp. 1320-1324, may 2000. [Online]. Available: 10.1016/s00223093(99)00944-8.

[16] N. Munzenrieder et al., "Flexible a-IGZO TFT amplifier fabricated on a free standing polyimide foil operating at $1.2 \mathrm{MHz}$ while bent to a radius of $5 \mathrm{~mm}$," pp. 5.2.1-5.2.4, 2012, doi: 10.1109/iedm.2012.6478982.

[17] E. Ungersboeck, S. Dhar, G. Karlowatz, V. Sverdlov, H. Kosina, and S. Selberherr, "The Effect of General Strain on the Band Structure and Electron Mobility of Silicon," IEEE Transactions on Electron Devices, vol. 54, no. 9, pp. 2183-2190, 2007, doi: 10.1109/ted.2007.902880. 